\title{
Online Survey for Patient Outcomes on Hericium Erinaceous Mushroom
}

\author{
Noha Abd Al Kreem Younis Younis*
}

\section{Noha Abd Al Kreem Younis Younis*}

Al Balqa Applied University, Aqaba Universal College; Lecturer - master's degree in Clinical Pharmacy, JORDAN.

\section{Correspondence}

Noha Abd Al Kreem Younis Younis

Al Balqa Applied University, Aqaba

Universal College; Lecturer - master's degree in Clinical Pharmacy, JORDAN.

Phone no: 00962-797773201;

E-mail: younisnoha@bau.edu.jo

History

- Submission Date: 11-02-2020;

- Review completed: 29-02-2020;

- Accepted Date: 20-03-2020

DOI : 10.5530/pj.2020.12.79

Article Available online http://www.phcogj.com/v12/i3

Copyright

(c) 2020 Phcogj.Com. This is an openaccess article distributed under the terms of the Creative Commons Attribution 4.0 International license.

\section{ABSTRACT}

Hericium Erinaceous (Lion's Mane) mushroom is steadfast becoming one of the most widespread and familiar of medicinal mushrooms currently available worldwide, this could be due to its many surprising health benefits and it is the ability to treat various ailments. In our study, we connected a questionnaire to the largest internet portal for Hericium Erinaceous (H.Erinaceous) consumers to measure respondents' feedback. The questionnaire addresses the attitude and potential benefits of respondents towards H.Erinaceousintake by using a numeric rating scale (NRS),97respondents took part in our study. The most frequently used of $H$. Erinaceousin our sample were in the treatment of neural diseases, enhance memory and GI disorders. The benefits and satisfied outcomes were highest with participants who use $H$. Erinaceous in a dose of 2-3 tablets ( $3 \mathrm{~g})$ twice/day to improve memory and cognitive properties and lowest in the treatment of autism even with high doses. $69.9 \%$ of participants did not discuss consuming this mushroom with their physician. Objectives for the study to determine the prevalence and purposes of using the mushroom in different diseases and to predict the outcomes, side effects and the most effective dose taken by participants.

Key words: Lion's Mane, Hericium Erinaceous, Medicinal mushrooms, Numeric rating scale.

\section{INTRODUCTION}

Mushrooms sit between plants and animals within the tree of life. Mushrooms are used as each food and drugs by humans for thousands of years. There are 14,000 species of mushrooms, which has roughly 2000 edible mushrooms, and two hundred mushrooms with potential as practical foods.

Lion's Mane mushroom is one in all these two hundred mushrooms. Lion's Mane mushroom is a palatable edible fungus that, has a taste like a prawn or scallop when cooked. In Japan, it is known as Yamabushitake, in China as Hou Tou Gu and as Hericium Erinaceous in Latin. It's a protracted history of use in ancient Chinese drugs and their extracts often used in over-the-counter health supplements found in forms of Capsule, Liquid, Tablet, and Powder.

Mushrooms secrete extracellular enzymes that permit them to assimilate nutrients by the absorption of the straightforward molecules that are created following the accelerator degradation method. Mushrooms are naturally made in proteins, fiber, carbohydrates, vitamins, and minerals and are low in calories and fats, particularly steroid alcohol.

H.Erinaceous mushroom contains over seventy bioactive compounds, the most important are hericernones, erincines, hericerins, resorcinols, steroids, and mono- and diterpenes. ${ }^{1}$

The sugar content of Lion's Mane mushroom is preponderantly aldohexose (68\%), arabinose (11\%) and other sugar $(7.8 \%)$.

Analytical information is simply offered on one Lion's Mane product on the North American country Food Composition info web site. ${ }^{2}$ This analysis shows that it contains $167 \mathrm{kcal}$ and thirty $3.3 \mathrm{~g}$ of sugar (per one hundred $\mathrm{g}$ of Lion's Mane mushroom).

Chinese medicine, as well as Japanese medicine, had a prolonged history of using this mushroom to help treat various ailments. It is used to treat gastric ulcer sand to improve digestion as well as ameliorate overall health by tonifying the main internal organs (lungs, liver, kidneys, heart, spleen,), and there are more than 7000 Lion's Mane-allied products available on Amazon for sale.

More than eleven papers are revealed on the Lion's Mane discuss the role of the lion's mane on aggression and sexuality, ${ }^{3}$ and many papers have been published in the role of H.Erinaceous in different medical purposes. Most of these studies are on the animal and cell line model, however very little were clinical trials on the human that are available online.

\section{MATERIAL AND METHODS}

In our study, we post a standardized online questionnaire on the social networking website. The questionnaire was a multiple-choice question in which we ask the participants to describe their attitude towards lion mane mushroom. Patients asked whether they use $H$. Erinaceous and if so, in which condition they were used, whether they communicated this to their physician, followed by questions about dose, frequency, and duration of consuming the mushroom. Next, we measured their satisfaction of outcomes using numeric rating scales (NRS)from 0 to 10 (zero is no benefit (unsatisfied 
with outcomes) and 10 is a full cure (satisfied with outcomes) and side effects that they may experience. Finally, if they advise other people to take this mushroom. Our objective was to investigate the prevalence, satisfaction and feedback of $H$. Erinaceous usage in different diseases and different doses. An online questionnaire was programmed using 'Access', from 1st July 2019 to 31st September 2019. Statistics were done by using IBM SPSS Statistics 20 (IBM Corp., Armonk, NY, USA).

\section{RESULTS}

A total of 100 participants answered the questions. Of these, three excluded because they didn't answer all questions, 34 currently received 1 or more types of conventional therapies in combination with lion's mane mushroom.

A total of 93 patients currently used $H$. Erinaceous, among the $H$. Erinaceous users, $30 \%$ of participants (28/97) had talked about $H$. Erinaceous with their physician, 69.9\% (65/97) had not Table 1 . Clarify that $77.8 \%$ of $H$. Erinaceous users were more than 40 years ( $(26.8 \%$ users were in the age of (41-50 years), $33.0 \%$ were in the age of (30-40years), $17.5 \%$ more than 50 years, $14.4 \%$ were between $21-30$ years)) and about $4.1 \%$ were in the age of (7-12) years old. (78.4\%) of our participants (76/97) were females.

Figure 1 shows which condition that the participants were using $H$. Erinaceous. About 23.7\% (23/97) of participants being treated with $\mathrm{H}$. Erinaceous for nervous system diseases, Alzheimer and to regenerate neural cells. And 17.5\% (17/97) of our sample used it for improving memory and cognitive function. About 16.5\% (16/97) used this mushroom in the treatment of GIS diseases especially peptic ulcer and a small proportion of participants used it to treat Autism (4.1\%).

The majority of participants $(52.6 \%)$ took this mushroom in a dose of 2-3 tablets (3gram) twice daily (59.8\%) as stated in Table 2 in details. Most of our sample used $H$. Erinaceous for 1-3 months. The major advisor to take this kind of mushroom was relatives and friends (77.8\%), and about (20\%) from different social media.

Finally, we analyzed whether patients were satisfied with the benefits and outcomes of the mushroom that they used as presented in Table 3, the original scale used is Numeric rating scale (NRS) with ratings from 0 to 10 , very satisfied (cure) $(=10)$ to not at all satisfied (no benefit) $(=0)$. The results show a satisfaction level of 10 more than $9.1 \%(9 / 97)$, about $30.9 \%(30 / 97)$ of participants satisfied with level from 1 to 5 , about $58.71 \%$ (57/97) satisfied with level from 6 to 9 and 10.3\% (10/97) show a satisfaction level of zero(not satisfied). The benefits and satisfied outcomes were highest with participants who use H. Erinaceous in a dose of 2-3 tablets ( $3 \mathrm{~g}$ ) twice/day to improve memory and cognitive properties. Satisfaction is lowest with patients complained of autism (4.1\%) (4/97) even with high doses of 5-6 tablets twice-three/day.

Table 1: The age and gender of $\boldsymbol{H}$. Erinaceous participants.

\begin{tabular}{cccc}
\hline & & Frequency & Percent \\
\hline \multirow{4}{*}{ Age } & 3-6 years & 1 & 1.0 \\
& 7-12 years & 4 & 4.1 \\
& $13-20$ years & 3 & 3.1 \\
& $21-30$ years & 14 & 14.4 \\
& 31-40 years & 32 & 33.0 \\
& $41-50$ years & 26 & 26.8 \\
& $>50$ years & 17 & 17.5 \\
& Total & 97 & 100.0 \\
& male & 21 & 21.6 \\
& female & 76 & 78.4 \\
& Total & 97 & 100.0 \\
\hline
\end{tabular}

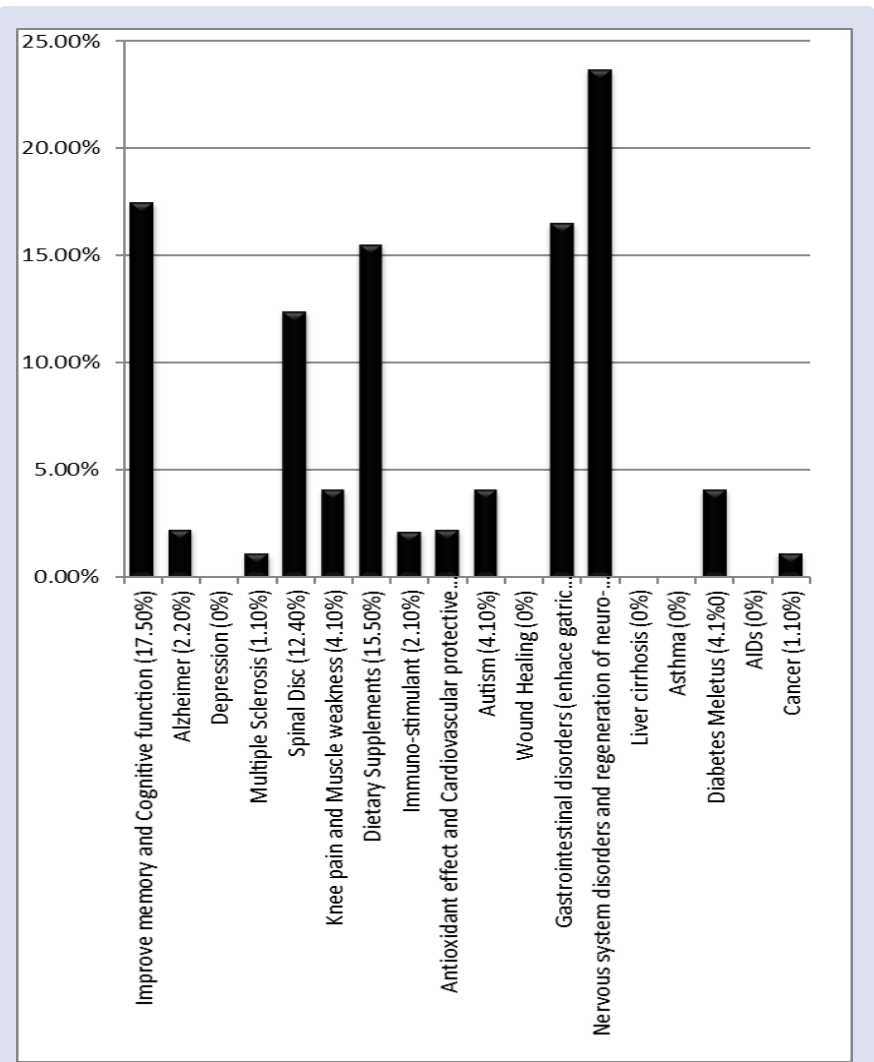

Figure 1: Conditions and diseases that the participants were using $H$. Erinaceous.

Table 2: The Dose and frequency of $\boldsymbol{H}$. Erinaceous intake.

\begin{tabular}{cccc}
\hline & & Frequency & Percent \\
\hline \multirow{5}{*}{ Dose } & 2-3 tablets(3gram)/day & 51 & 52.6 \\
& 3-4 tablets(4gram)/day & 22 & 22.7 \\
& $4-5$ tablets(5gram)/day & 8 & 8.2 \\
& $5-6$ tablets/day & 16 & 16.5 \\
Frequency & Total & 97 & 100.0 \\
& Once daily & 30 & 30.9 \\
& Twice daily & 58 & 59.8 \\
& Three times daily & 9 & 9.3 \\
\hline
\end{tabular}

Table 3: Satisfaction (benefits) outcomes of consuming H. Erinaceous.

\begin{tabular}{cccc}
\hline & & Frequency & Percent \\
\hline & $\begin{array}{c}\text { Unsatisfied (No } \\
\text { benefit) }\end{array}$ & 10 & 10.3 \\
& 1 & 2 & 2.1 \\
& 2 & 3 & 3.1 \\
Benefit & 3 & 2 & 2.1 \\
(outcome)s & 5 & 7 & 7.2 \\
& 5 & 16 & 16.5 \\
& 7 & 17 & 17.5 \\
& 8 & 14 & 14.4 \\
& 9 & 13 & 13.4 \\
& 10 Satisfied (cured) & & \\
\hline
\end{tabular}

The majority of $H$. Erinaceous users (86.6\%) (84/97) were not experienced any side effects as stated in Table 4 after taking the mushroom regularly for 3-6 months and (97.9\%) (95/97) advise others to intake the mushroom. 
Table 4: Side effects of $\boldsymbol{H}$. Erinaceous.

\begin{tabular}{lccc}
\hline & & Frequency & Percent \\
\hline \multirow{3}{*}{ Side Effects } & No & 84 & 86.6 \\
& Yes & 13 & 13.4 \\
& Allergy & 2 & 2.1 \\
Symptoms & Rash & 2 & 2.1 \\
& Gastric upset & 2 & 2.1 \\
& Diarrhea & 5 & 5.2 \\
& Difficult of breathing & 1 & 1.0 \\
\hline
\end{tabular}

\section{DISCUSSION}

It's not simply that there are restricted clinical studies on $H$. Erinaceous mushroom. A comprehensive review of Lion's Mane mushroom revealed in 2015 delineated the knowledge on Lion's Mane mushroom as being "widely scattered".

\section{The role of lion's mane mushroom in cognitive function}

It is attainable that lion's mane mushrooms would possibly boost cognitive function, however, the prevailing analysis is especially on animals but its scientific proof for human health still uncertain completely.

A double-blind, randomized, comparative study conducted in Japan in 2019 assessed the improvement of the cognitive functions by 12 weeks intake of $H$. erinaceous supplements. This study confirmed that this mushroom had potential effects on the neural networks of the brain and can improve cognitive functions. Moreover, confirm the safety of H.erinaceous and could be used in dementia prevention. ${ }^{5}$ Another recent Italian study in 2019 on mice reported that two-month of oral intake of H.erinaceous reversed the decline of age and restore cognition memory, which means this mushroom could have neurogenesis effect. ${ }^{6}$ In line with a vitro study conducted in Malaysia showed that Lion's Mane mushrooms contain neurotrophic factors (hericenones and erinacines) which might induce nerve growth factors essential for the maintenance of optimum neural body structure to operate. In summary, the full study showed that Lion's Mane mushroom had neurotrophic (meaning growth of neuron tissue) but no neuroprotective effects. All these findings match our results that the intake of a lion's mane for more than 3 months can improve cognitive properties without any side effects noted in participants.

\section{The role of lion's mane mushroom mane in inflammation}

Lion's Mane mushroom has an anti-inflammatory effect due to its capability to decrease Nitric oxide production which works as a proinflammatory mediator.

There are many pieces of research studying Lion's Mane mushroom in inflammation, however, no clinical studies.

In most recent studies conducted in Taiwan in 2019 assessed the antineuroinflammatory effect of $H$. erinaceous activation of microglial cells that can be induced by LPS, which produces large amounts of reactive and demonstrate the possible mechanism of action by using human BV2 microglial cells induced by LPS. ${ }^{8}$ The same findings in a study performed in china showed that the extract of Lion's Mane mushrooms exhibited anti-inflammatory activity due to the suppression of nitric oxide generation, suggesting a doable role for Lion's Mane mushroom as either a preventive or therapeutic anti-inflammatory agent. ${ }^{9}$ In 2015 a research was conducted in Japan indicate that lion mane extract can exert anti-inflammatory effects on macrophages by inhibiting JNK signaling and avoid the inflammation of adipose tissue that related to obesity. ${ }^{10}$

Another research conducted on mice in 2016 found that the ethanol lion mane extract has anti-inflammatory effects in ulcerative colitis mice and can be used in the treatment of IBDs, by inhibiting the production of different inflammatory mediators in gastrointestinal tissue including interleukin (IL)- $1 \beta$, tumor necrosis factor- $\alpha$ and IL- 6 , and can regulate the production of different substances that play important role in oxidative stress such as nitric oxide and superoxide dismutase. ${ }^{11}$

\section{The role of lion's mane mushroom mane in depression} and anxiety

There are many causes of depression and anxiety and chronic inflammation could be the major contributing factor, so antiinflammatory substances considered to have anti-depressant effects. Which could have been another factor in the reduction of depression.

Studies suggest that H.Erinaceous mushrooms can treat mild symptoms of anxiety and depression, one of these studies was an animal study conducted in Japan in 2015 found that the Amycenone that obtained from the extracts of lion's mane mushroom has anti-depressant effects in mice model by inhibiting the inflammation-related depression. ${ }^{12}$

A study conducted on stroke rat model (SAM), showed that rats who were given an extract made from Lion's Mane exhibit reduction in the total infarcted volumes by $44 \%$ and the levels of acute inflammatory response were decreased by Erinacine A. Scientists attribute this effect to the active substances found in mushrooms specifically the hericenones and erinacines which could in the future play a role in treating depression as well as anxiety. ${ }^{13}$

The findings of a 2018 animal study support this, with the authors concluding that this mushroom extract may contain agents that Modulate BDNF/PI3K/Akt/GSK-3 $\beta$ Signaling in Mice that are useful for treating depressive disorders. ${ }^{14}$

\section{The role of lion mane mushroom mane in alzheimer's} disease

The formation of amyloid plaques is considered to be the morphological biomarker associated with Alzheimer's disease. These plaques form when a protein called beta-amyloid (BAY-tuh AM-uh-loyd) cluster together. Beta-amyloid comes from another protein exists in the fatty membrane surrounding the nerve cells. Beta-amyloids clump gradually and build up into plaques. These clumps may block signal neuron transmission.

A recent survey conducted on induced memory loss mice due to injected the mice with neurotoxic peptides then fed these mice a diet containing 5\% Lion's Mane mushroom, the result confirmed that the damaging amyloid plaques were significantly decreased, and improve in the memory in Alzheimer mice by using a maze memory test. ${ }^{15}$ It is the ability to regrow Myelin the fatty substance around nerve cells this mushroom could be very helpful in treating degenerative nerve conditions in the future. ${ }^{16}$

Other researchers have concluded that the mushrooms may have the potential to treat or prevent diseases that cause a decline in cognitive health, such as Parkinson's disease and Alzheimer's disease. The Neuroprotective Properties of H.Erinaceusin Glutamate-Damaged Differentiated PC12 Cells associated an Alzheimer's malady Mouse Model, however, there is currently a lack of research on the effects of lion's mane mushrooms in humans with Alzheimer's disease. ${ }^{17}$

The role of lion's mane mushroom mane in diabetes meletus

\section{Controlling blood sugar levels is the key to managing diabetes}

One study showed that blood sugar levels in rats with diabetes were lower after they received a lion's mane mushroom extract for 4 weeks and a significant increase in serum insulin level. ${ }^{18}$ 
One of the complications of diabetes is nerve damage resulting from prolonged periods of high blood sugar. A 2015 study on rats, in which they ingested lion's mane extract for 6 weeks, showed positive results, including lower blood sugar levels, reduced feelings of nerve pain, and improved antioxidant activity. ${ }^{19}$

Another study carried out in 2018 used fermented H.Erinaceus juice (FHJ) in male Wistar rats with streptozotocin-induced diabetes mellitus (DM). The study confirmed that fermented Herinaceus juice may be used as one of the food-based health-promoting supplements to treat DM along with medication..$^{20,21}$

\section{The role of lion's mane mushroom mane in cardiovascular diseases and cholesterol}

Lion's mane extract could improve heart health, however, the analysis to this point has primarily used animal subjects.

Italian study conducted in 2019 on Seventy-seven obese volunteers, documented that supplementation of oral $H$. erinaceous for eight weeks could decrease obesity and other disorders associated with obesity. ${ }^{22}$

A second study also done in Japan found that $H$. erinaceous mushrooms significantly reduced lipid plasma levels in mice that were fed a highfat diet. laboratory tests, including total cholesterol (TC), triglyceride (TG) levels and low-density lipoprotein-cholesterol (LDL-c) decreased in the rats given the Lion's Mane extract. ${ }^{23}$ Taken along, these 2 studies raise the chance that Lion's Mane mushrooms play a role in preventing cardiopathy and indicates that this mushroom can be used in the future for the treatment of hyperlipidemia in humans. this can be potential however, faraway from established at now. it's an extended hop from bunny rabbits and mice to human health.

\section{The role of lion's mane mushroom mane in cancer}

There are twenty-four publications concerning cancer and Lion's Mane mushrooms. sadly, none of those involve human subjects. The newest study conducted in Pakistan studied the anti-proliferative effect of $H$. Erinaceus and other commercial mushrooms on cell line model, it confirmed the Anticancer, antithrombotic, anti-tyrosinase, and anti- $\alpha$ glucosidase activities of this mushroom. ${ }^{24}$

In 2018 A Chinese study reported that erinacine which extracted from $H$. Erinaceus can promote apoptosis and inhibited cell proliferation by inducing MPTP opening and promote the release of cyt-C which plays a role in inhibiting the progression of Hepatocellular Carcinoma. ${ }^{25}$ Further research showed that the extracts of $H$. Erinaceus given orally, have proved a tumor-suppressing activity similar to that of 5 -fluorouracil, in the treatment of gastrointestinal cancers. ${ }^{26}$

Other research in cancer cell lines confirmed the potential role of Lion's Mane mushrooms as an antineoplastic agent, either alone or together with other therapeutical agents. ${ }^{27}$

\section{The role of lion mane mushroom mane in gastro- intestinal diseases}

Lion's mane might facilitate digestive health by fighting inflammation, that might be helpful for individuals with inflammatory bowel disease (IBD). The mushroom may additionally boost immune function and promote the growth of beneficial bacteria within the gut. A study performed in the USA utilizes the effect of $H$. erinaceous in Vitro and in Vivo on Helicobacter pylori and they confirmed that IL-8 production as an immune response to $H$. pylori was significantly decreased after giving $H$. erinaceous extract at a concentration of $1.0 \mathrm{mg} / \mathrm{mL}$ extract. ${ }^{28}$

Another new Chinese study conducted in 2019 used cellular injury model induced by deoxynivalenol (DON)suggested that In Vitro and
In Vivo Inhibition of Helicobacter pylori by Ethanolic Extracts of Lion's Mane Medicinal Mushroom, and might significantly protect mucosal cells from oxidative stress and decrease the formation of reactive oxygen species the induced by (DON) $)^{29,30} \mathrm{~A}$ similar study conducted in china recently on mice proofed that the polysaccharide extracted from $H$. Erinaceous had a potent effect as anti-Ulcerative Colitis. ${ }^{31}$ Further studies assessed the efficacy of $\mathrm{H}$. Erinaceous extract in Helicobacter pylori activity, ${ }^{32}$ chronic gastritis, ${ }^{33}$ IBD. ${ }^{34}$

Our study showed good outcomes after intake of H.Erinaceous supplements in patients with different gastrointestinal diseases, and the satisfaction of participants was 6 from 10 (18.8\%).

The role of lion's mane mushroom in the immunity system

Lion's mane mushrooms may enhance the immune system by reducing inflammation and preventing oxidation.

A recent study performed in China-2019 reported that the macromolecular $\beta$-glucan H6PC20 obtained from $H$. Erinaceous had potential bioactivity in enhancing immunity, activating the proliferation of lymphocytes and promote the expression of inflammatory cytokines that secreted by macrophages. ${ }^{35}$

\section{The role of lion's mane mushroom in wound healing}

Extracts from lion's mane mushrooms could promote healing in case of skin wounds. A study on rats with neck wounds found that topical application of a lion's mane extract can enhance faster healing ${ }^{36}$ However, additional research is critical for the health profession to suggest lion's mane mushrooms for topical use on humans.

\section{The role of lion's mane mushroom in the nervous system}

Damage to the nervous system can have significant effects on health. Recently a new study published in Taiwan demonstrated that Glia cells especially Microglia which had an important role in the signal transduction pathways of chronic pain inhibit morphine-induced BV2 activations by regulating the deacetylation of signal transduction pathway of HDAC6/HSP90. ${ }^{37}$ Another study conducted in Malaysia in 2019 reported that $H$. Erinaceous could have a significant neuroprotective effect and anti-inflammatory effect in the neuronglia environment inH2O2-Induced Oxidative stress and LPS-Induced Inflammation in cell model. ${ }^{38}$ Older research suggests that extracts of lion's mane mushrooms may encourage nerve cells to grow and repair more quickly. ${ }^{39}$ These findings similar to our results that intake of lion's mane for more than 3 months can have neuroprotective effects and assist in the treatment of different neural diseases, feedback satisfaction of $H$. Erinaceous intake was (18.2\%) 7 out of 10 .

\section{The role of lion's mane mushroom mane as antioxidant}

A new study done on mice model in Taiwan suggested that erinacine That extracted from $H$. erinaceous might promote longevity by inducing endogenous antioxidants enzymes. ${ }^{40}$ Moreover, many other studies evaluated antioxidant activity in vitro and on rats model founded that $H$. erinaceous mushrooms had a significant role in vitro antioxidant action. ${ }^{41,42}$

\section{Safety and side effects of lion's mane mushroom}

Most studies on lion's mane mushrooms have used animals' models, however, it seems to be safe to eat the mushrooms in moderate quantities, as people in several countries in Asia do. The majority of participants $(52.6 \%)$ took this mushroom in a dose of 2-3 tablets (3gram) twice daily (59.8\%) 
Oral administration of doses of up to $1000 \mathrm{mg} / \mathrm{kg}$ daily divided into two to three doses for ninety days of $H$. Erinaceous was deemed to be effective and safe supported autopsy studies of sacrificed rats. ${ }^{43}$

A second study in Sprague-Dawley rats showed that doses of up to $3,000 \mathrm{mg} / \mathrm{kg} /$ day were safe supported autopsy samples once more. ${ }^{44}$

The safety and effectiveness of lion's mane supplements are not clear as a result of dietary supplements don't have a similar regulation as food and drug product. However, within the animal studies, one of them was newly published in Taiwan suggested that $H$. erinaceous might be comparatively unharmful when used over a prolonged period, even with high doses reach $2625 \mathrm{mg} / \mathrm{kg}$ did not cause adverse effects within the rodents..$^{43,45}$ In our study small proportion (13/97) of participants experienced side effects such as rash and diarrhea.

\section{CONCLUSION}

Most studies on lion's mane mushrooms have used animals and there are not enough clinical studies on human can confirm the results.

The benefits and satisfied outcomes were highest with participants who use H. Erinaceous in a dose of 2-3 tablets twice/day to improve memory and cognitive properties in a period of more than 3 months. Satisfaction is not present (zero from 10) with patients complained of autism (4.1\%) (4/97) even with high doses of 5-6 tablets twice-three/day.

Additional studies required to accentual the efficacy and side effects in short-term and long-term therapy.

\section{DISCLOSURE STATEMENT}

No conflict of interest.

\section{REFERENCES}

1. Rebecca.p@bulletproof.com. "Lion's Mane Mushroom Benefits: Boost Memory, Focus \& Mood With This Nootropic." Bulletproof, December 12, 2018[ cited 2019 Nov 5];360(6)

2. Ndb.nal.usda.gov, ndb.nal.usda.gov/.

3. West, Peyton M, and Craig Packer. "Sexual Selection, Temperature, and the Lion's Mane." Science (New York, N.Y.), U.S. National Library of Medicine, 2002 Aug 23;297(5585):1339-43. Available from: www.ncbi.nlm.nih.gov/ pubmed/12193785.

4. "Chemistry, Nutrition, and Health-Promoting Properties of Hericium Erinaceus (Lion's Mane) Mushroom Fruiting Bodies and Mycelia and Their Bioactive Compounds." Journal of Agricultural and Food Chemistry, 2015 Aug;63(32):71087123. Available from: pubs.acs.org/doi/10.1021/acs.jafc.5b02914.

5. Saitsu, Yuusuke, et al. "Improvement of Cognitive Functions by Oral Intake of Hericium Erinaceus." Biomedical Research (Tokyo, Japan), U.S. National Library of Medicine, 2019;40(4):125-131. Available from: www.ncbi.nlm.nih. gov/pubmed/31413233

6. Ratto, Daniela, et al. "Hericium Erinaceus Improves Recognition Memory and Induces Hippocampal and Cerebellar Neurogenesis in Frail Mice during Aging." Nutrients, 2019 Mar 27;11(4):715. Available from: https://www.ncbi.nlm.nih. gov/pubmed/30934760

7. Lai, Puei-Lene, et al. "Neurotrophic Properties of the Lions Mane Medicinal Mushroom, Hericium Erinaceus (Higher Basidiomycetes) from Malaysia." International Journal of Medicinal Mushrooms, 2013;15(6):539-554. Available from: https://www.ncbi.nlm.nih.gov/pubmed/24266378

8. Wang, Li-Yu, et al. "Anti-Inflammatory Effect of Erinacine $\mathrm{C}$ on NO Production Through Down-Regulation of NF-KB and Activation of Nrf2-Mediated HO-1 in BV2 Microglial Cells Treated with LPS." Molecules, 2019 Sep 12;24(18):3317. Available from: https://www.ncbi.nlm.nih.gov/pubmed/31547327.

9. Geng, Yan, et al. "Anti-Inflammatory Activity of Mycelial Extracts from Medicinal Mushrooms." International Journal of Medicinal Mushrooms, 2014:16(4):319_ 325. Available from: https://www.ncbi.nlm.nih.gov/pubmed/25271860.

10. Mori, Koichiro, et al. "The Anti-Inflammatory Effects of Lions Mane CulinaryMedicinal Mushroom, Hericium Erinaceus (Higher Basidiomycetes) in a Coculture System of 3T3-L1 Adipocytes and RAW264 Macrophages." International Journal of Medicinal Mushrooms, 2015;17(7):609-18. Available from: https://www.ncbi.nlm.nih.gov/pubmed/26559695.

11. Yao, Wei, et al. "Effects of Amycenone on Serum Levels of Tumor Necrosis Factor- $\alpha$, Interleukin-10, and Depression-like Behavior in Mice after Lipopolysaccharide Administration." Pharmacology Biochemistry and Behavior, 2015 Sep;136:7-12. Available from: https://www.ncbi.nlm.nih.gov/ pubmed/26150007.

12. Yao, Wei, et al. "Effects of Amycenone on Serum Levels of Tumor Necrosis Factor- $\alpha$, Interleukin-10, and Depression-like Behavior in Mice after Lipopolysaccharide Administration." Pharmacology Biochemistry and Behavior, $2015 \mathrm{Jul}$ 4vol;136:7-12.

13. Li, I-Chen, et al. "Neurohealth Properties OfHericium ErinaceusMycelia Enriched with Erinacines." Behavioural Neurology,2018 May 21;2018:1-10. Available from: doi:10.1155/2018/5802634.

14. Chiu, Chun-Hung, et al. "Erinacine A-Enriched Hericium Erinaceus Mycelium Produces Antidepressant-Like Effects through Modulating BDNF/PI3K/Akt/GSK $3 \beta$ Signaling in Mice." International Journal of Molecular Sciences, 2018 Jan 24;19(2):341. Available from: https://www.ncbi.nlm.nih.gov/pubmed/29364170.

15. Sutherland, et al. "Herbal Medicine for Alzheimer's Disease: Lion's Mane (Hericium Erinaceus)." Restorative Medicine, 2018 Dec;6:19. Available from: restorativemedicine.org/journal/neurological-activity-lions-mane-hericiumerinaceus/.

16. Kushairi, Naufal, et al. "Lion's Mane Mushroom, Hericium Erinaceus (Bull.: Fr.) Pers. Suppresses H2O2-Induced Oxidative Damage and LPS-Induced Inflammation in HT22 Hippocampal Neurons and BV2 Microglia." Antioxidants (Basel, Switzerland), MDPI, 2019 Aug 1;8(8). Available from: www.ncbi.nlm.nih. gov/pubmed/31374912.

17. Zhang, Junrong, et al. "The Neuroprotective Properties of Hericium Erinaceus in Glutamate-Damaged Differentiated PC12 Cells and an Alzheimer's Disease Mouse Model." International Journal of Molecular Sciences, MDPI, 2016 Nov 1 17(11): 1810. Available from: www.ncbi.nlm.nih.gov/pmc/articles/PMC5133811/

18. Liang, Bin, et al. "Antihyperglycemic and Antihyperlipidemic Activities of Aqueous Extract of Hericium Erinaceus in Experimental Diabetic Rats." BMC Complementary and Alternative Medicine, BioMed Central, 2013 Oct 3;13:253. Available from: www.ncbi.nlm.nih.gov/pmc/articles/PMC3852124/.

19. Yi, Zhang, et al. "Protective Effect of Ethanol Extracts of Hericium Erinaceus on Alloxan-Induced Diabetic Neuropathic Pain in Rats." Evidence-Based Complementary and Alternative Medicine : ECAM, Hindawi Publishing Corporation, 2015 April 16;2015:5. Available from: www.ncbi.nlm.nih.gov/pmc/ articles/PMC4415746/.

20. Chaiyasut, Chaiyavat, et al. "Lactobacillus Fermentum HP3-Mediated Fermented Hericium Erinaceus Juice as a Health Promoting Food Supplement to Manage Diabetes Mellitus." Journal of Evidence-Based Integrative Medicine, SAGE Publications, 2018 Jan-Dec;23:2515690X18765699. Available from: www.ncbi.nlm.nih.gov/pmc/articles/PMC5894895/.

21. Zhang, Chen, et al. "Antihyperglycaemic and Organic Protective Effects on Pancreas, Liver and Kidney by Polysaccharides from Hericium Erinaceus SG-02 in Streptozotocin-Induced Diabetic Mice." Scientific Reports, Nature Publishing Group UK, 2017 Sep 7;7(1):10847. Available from: www.ncbi.nlm.nih.gov/pmc/ articles/PMC5589823/.

22. Vigna, Luisella, et al. "Hericium Erinaceus Improves Mood and Sleep Disorders in Patients Affected by Overweight or Obesity: Could Circulating Pro-BDNF and BDNF Be Potential Biomarkers?" Evidence-Based Complementary and Alternative Medicine : ECAM, Hindawi, 2019 Apr 18;2019:7861297. Available from: www.ncbi.nlm.nih.gov/pubmed/31118969.

23. Choi, Won-Sik, et al. "Hypolipidaemic Effect of Hericium Erinaceum Grown in Artemisia Capillaris on Obese Rats." Mycobiology, The Korean Society of Mycology, June 2013, www.ncbi.nlm.nih.gov/pmc/articles/PMC3714447/.

24. Sharif, Sumaira, et al. "Anticancer, Antithrombotic, Antityrosinase, and Anti$\alpha$-Glucosidase Activities of Selected Wild and Commercial Mushrooms from Pakistan." Food Science \& Nutrition, John Wiley and Sons Inc., 2018 Sep 14;6(8):2170-2176. Available from: www.ncbi.nlm.nih.gov/pubmed/30510718.

25. Zhou, Li-Jie, et al. Erinacine Facilitates the Opening of the Mitochondrial Permeability Transition Pore Through the Inhibition of the PI3K/ Akt/GSK-3 $\beta$ Signaling Pathway in Human Hepatocellular Carcinoma 2018:50(3):851-867. Available from: www.ncbi.nlm.nih.gov/pubmed/30355923.

26. Li, Guang, et al. "Anticancer Potential of Hericium Erinaceus Extracts against Human Gastrointestinal Cancers." Journal of Ethnopharmacology, 2014 Apr 28;153(2):521-30. doi:10.1016/j.jep.2014.03.003.

27. Li, Guang, et al. "Anticancer Potential of Hericium Erinaceus Extracts against Human Gastrointestinal Cancers." Journal of Ethnopharmacology, U.S. National Library of Medicine, 2014 Apr 28;153(2):521-30. Available from: www.ncbi.nlm. nih.gov/pubmed/24631140.

28. Wang, Ge, et al. "In Vitro and In Vivo Inhibition of Helicobacter Pylori by Ethanolic Extracts of Lion's Mane Medicinal Mushroom, Hericium Erinaceus (Agaricomycetes)." International Journal of Medicinal Mushrooms, Begel House Inc, 2018 January; 21(1). Available from: www.dl.begellhouse.com/journ als/708ae68d64b17c52,2806f82d786f0ca1,6e9ef24864bd9e52.html.

29. Qin, Tao, et al. "Characterization of Polysaccharides Isolated from Hericium Erinaceus and Their Protective Effects on the DON-Induced Oxidative Stress." International Journal of Biological Macromolecules, U.S. National Library of Medicine, 2019 Nov 20. pii: S0141-8130(19)37739-6. 
30. Yeh, Chung-Hsin et al "Effect of Ethanol Extracts of Hericium Erinaceus Mycelium on Morphine-Induced Microglial Migration." Molecular Medicine Reports, U.S. National Library of Medicine, 2019 October 15:20(6):5279-5285.

31. Shao, Shuai, et al. "A Unique Polysaccharide from Hericium Erinaceus Mycelium Ameliorates Acetic Acid-Induced Ulcerative Colitis Rats by Modulating the Composition of the Gut Microbiota, Short Chain Fatty Acids Levels and GPR41/43 Respectors." International Immunopharmacology, U.S. National Library of Medicine, 2019 Jun;71:411-422. Available from: www.ncbi.nlm.nih. gov/pubmed/31059977.

32. Liu, Jian-Hui, et al. "Anti-Helicobacter Pylori Activity of Bioactive Components Isolated from Hericium Erinaceus." Journal of Ethnopharmacology, U.S. National Library of Medicine, 2016 May 13;183:54-58.

33. Wang, Mingxing, et al. "A Polysaccharide from Cultured Mycelium of Hericium Erinaceus and Its Anti-Chronic Atrophic Gastritis Activity." International Journal of Biological Macromolecules, U.S. National Library of Medicine, 2015 Nov;81:656-61. Available from: www.ncbi.nlm.nih.gov/pubmed/26314904.

34. Diling, Chen, et al. "Extracts from Hericium Erinaceus Relieve Inflammatory Bowel Disease by Regulating Immunity and Gut Microbiota." Oncotarget, Impact Journals LLC, 2017 Sep 6;8(49):85838-85857.

35. Wu, Di, et al. "Structural Elucidation and Immunomodulatory Activity of a $\beta$-D Glucan Prepared by Freeze-Thawing from Hericium Erinaceus." Carbohydrate Polymers, U.S. National Library of Medicine, 2019 Oct 15:222:114996.

36. Abdulla, Mahmood Ameen, et al. "Potential Activity of Aqueous Extract of Culinary-Medicinal Lion's Mane Mushroom, Hericium Erinaceus (Bull.: Fr.) Pers. (Aphyllophoromycetideae) in Accelerating Wound Healing in Rats." International Journal of Medicinal Mushrooms, U.S. National Library of Medicine, 2011;13(1):33-9.

37. Yeh, Chung-Hsin, et al. "Effect of Ethanol Extracts of Hericium Erinaceus Mycelium on Morphine-Induced Microglial Migration." Molecular Medicine Reports, U.S. National Library of Medicine, 2019 Oct 15;20(6): 5279-5285.
38. Kushairi, Naufal et al "Lion's Mane Mushroom Hericium Erinaceus (Bull. Fr.) Pers. Suppresses H2O2-Induced Oxidative Damage and LPS-Induced Inflammation in HT22 Hippocampal Neurons and BV2 Microglia." Antioxidants (Basel, Switzerland), MDPI, 2019 Aug 1;8(8). pii: E261.

39. Samberkar, Snehlata, et al. "Lion's Mane, Hericium Erinaceus and Tige Milk, Lignosus Rhinocerotis (Higher Basidiomycetes) Medicinal Mushrooms Stimulate Neurite Outgrowth in Dissociated Cells of Brain. Spinal Cord, and Retina: An In Vitro Study." International Journal of Medicinal Mushrooms, U.S National Library of Medicine, 2015;17(11):1047-54.

40. Li, I-Chen, et al. "Erinacine A-Enriched Hericium Erinaceus Mycelia Promotes Longevity in Drosophila Melanogaster and Aged Mice." PloS One, Public Library of Science, 2019 may 17; 14(5): e0217226.

41. Chen, Juan, et al. "Genomic and Transcriptomic Analyses Reveal Differentia Regulation of Diverse Terpenoid and Polyketides Secondary Metabolites in Hericium Erinaceus." Scientific Reports, Nature Publishing Group UK, 2017 Aug 31;7(1):10151.www.ncbi.nlm.nih.gov/pubmed/28860534.

42. Hou, Yiling, e t al. "Composition and Antioxidant Activity of Water-Soluble Oligosaccharides from Hericium Erinaceus." Molecular Medicine Reports, U.S. National Library of Medicine, Available from: www.ncbi.nlm.nih.gov/ pubmed/25529054.

43. Lakshmanan, Hariprasath, et al. "Haematological, Biochemical and Histopathological Aspects of Hericium Erinaceus Ingestion in a Rodent Model: A Sub-Chronic Toxicological Assessment." Journal of Ethnopharmacology, 2016 Dec 24;194:1051-1059.

44. Li, I-Chen et al. "Evaluation of the Toxicological Safety of Erinacine A-Enriched Hericium Erinaceus in a 28-Day Oral Feeding Study in Sprague-Dawley Rats." Food and Chemical Toxicology : an International Journal Published for the British Industrial Biological Research Association, U.S. National Library of Medicine 2014 Aug;70:61-7.

45. Lee, Li-Ya, et al. "Thirteen-Week Oral Toxicity Evaluation of Erinacine AEnriched Lion's Mane Medicinal Mushroom, Hericium Erinaceus (Agaricomycetes), Mycelia in Sprague-Dawley Rats." International Journal of Medicinal Mushrooms, U.S. National Library of Medicine, 2019 Jan 01, 21(4):401-411,

\section{GRAPHICAL ABSTRACT}

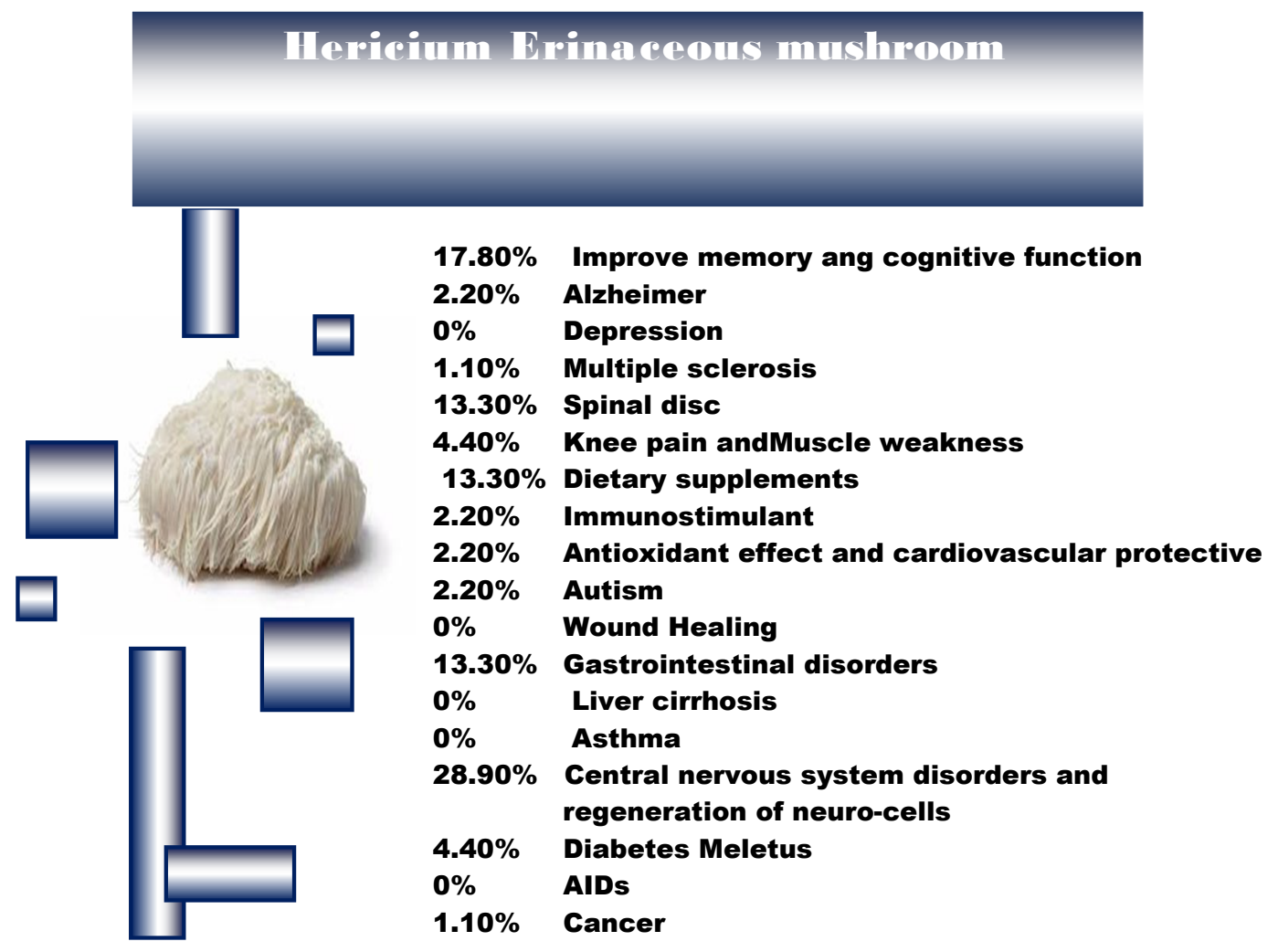




\section{ABOUT AUTHORS}

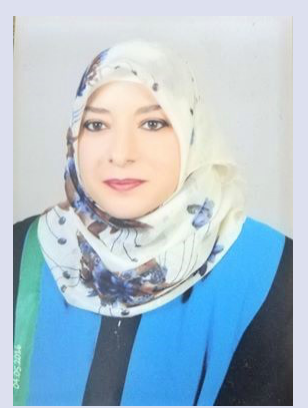

Noha Abd Al Kreem Younis Younis (Y.Noha)

Al Balqa Applied University - Aqaba Universal College; Lecturer - master's degree in Clinical Pharmacy, Jordan.

Cite this article: Younis NAAKY. Online Survey for Patient Outcomes on Hericium Erinaceous Mushroom. Pharmacogn J. 2020;12(3):519-25. 